\title{
La autopercepción del apoyo social del paciente con enfermedad crónica, mediado por tecnología*
}

Self-Perception of Technology-Aided Social Support in Patients with Chronic Diseases

A autopercepção do apoio social do paciente com doença crônica, mediado pela tecnologia

Adriana Hernández Bustos ${ }^{a}$

Universidad de Cundinamarca, Colombia

DOI: https://doi.org/10.11144/Javeriana.ie21-2.aasp

ahernandezb@ucundinamarca.edu.co

ORCID: http://orcid.org/0000-0001-8146-5413

Maria Victoria Rojas Martinez

Universidad de Cundinamarca, Colombia

ORCID: http://orcid.org/0000-0003-1637-4800

Fecha de recepción: 17 Julio 2018

Fecha de aprobación: 14 Junio 2019

Gloria Naidú Cuevas Rodriguez

Universidad de Cundinamarca, Colombia

ORCID: http://orcid.org/0000-0002-6403-4782

Fecha de publicación: 30 Noviembre 2019

\section{Resumen:}

Introdución: el apoyo social ha sido un componente fundamental para el cuidado de la persona con enfermedad crónica; por lo tanto, conocer la percepción que se tiene de este y cómo la tecnología es un medio para fortalecerlo es relevante en la formulación de los planteamientos de cuidado que se implementan en las instituciones de salud. Objetivo: determinar el efecto de la intervención Alguien apoya al que cuida en las percepciones de apoyo social que tienen los enfermos crónicos de un servicio de consulta externa en una institución pública de Girardot (Cundinamarca, Colombia). Método: estudio de tipo cuantitativo-preexperimental, con una muestra de 116 personas con enfermedad crónica. Se realizó una caracterización sociodemográfica, de condiciones para el cuidado y nivel de apropiación de las tecnologías de los pacientes usando la ficha Caracterización de la Persona con Enfermedad Crónica o GCPC-UN-P. Como estrategia se desarrollaron tres talleres aplicando la intervención Alguien apoya al que cuida al grupo experimental y se midió la percepción de apoyo social antes de la intervención y después de esta, utilizando el Cuestionario MOS de Soporte Social Percibido. Resultados: el efecto de la intervención fue estadísticamente significativo $(p<0,05)$ y cada uno de los factores que la componen: apoyo emocional, informacional, apoyo instrumental e interacción positiva afectiva. Conclusiones: las intervenciones con el uso de recursos audiovisuales son efectivas como apoyo para los pacientes; y aquí la televisión y la radio son los medios en los cuales se encuentra mayor apropiación en esta población.

Palabras clave: apoyo social, enfermedad crónica, tecnología, recursos audiovisuales.

\section{Abstract:}

Introduction: Social support has been an essential component essential in taking care of people with chronic diseases. Therefore, when planning the health care to be provided in the health centers it is important to know how the social support is perceived and how the technology is useful to strengthen it. Objective: To determine the effect of the intervention 'Supporting the Caregiver' on the social support perceptions by the chronic patients who attend to the outpatient services at a public health center in Girardot (Cundinamarca, Colombia). Methods: It is a quantitative pre-experimental study with a sample of 116 chronic-disease patients. Sociodemographic characteristics were outlined in relation to the care conditions and level of technology adoption by the patients, as measured with the instrument 'Characterization of a Patient with a Chronic Disease or GCPC-UN-P'. The strategy consisted in three workshops applying the intervention 'Supporting the Caregiver' to the experimental group and measuring the social support perception both before and after the intervention based on the MOS questionnaire for perceived social support. Results: The effect of the intervention was statistically significant ( $p$ \&lt; 0.05 ) as well as each factor making part of it: emotional support, informational support, instrumental support and affective positive interaction. Conclusions: Interventions with audiovisual resources are effective when providing support to these patients; the radio and television are the media most embraced among this population.

Notas de autor

${ }^{a}$ Autora de correspondencia. Correo electrónico: ahernandezb@ucundinamarca.edu.co 
Keywords: social support, chronic disease, technology, audiovisual resources.

\section{Resumo:}

Introdução: $\mathrm{O}$ apoio social tem sido um componente fundamental para o cuidado da pessoa com doença crónica; porém, conhecer a percepção que dele se tem e como a tecnologia é um médio para fortalecê-lo é relevante na formulação das abordagens de cuidado implementadas nas instituições de saúde. Objetivo: Determinar o efeito da intervenção Alguém apoia o cuidador nas percepções de apoio social que os doentes crônicos têm de um serviço de consulta externa em uma instituição pública de Girardot (Cundinamarca, Colômbia). Método: Estudo quantitativo-pré-experimental, com amostra de 116 pessoas com doença crônica. Uma caracterização sociodemográfica de condições para o cuidado e nível de apropriação das tecnologias dos pacientes foi feita usando o formato Caracterização da Pessoa com Doença Crônica ou GCPC-UN-P. Como estratégia três oficinas foram desenvolvidas aplicando a intervenção Alguém apoia o cuidador ao grupo experimental e foi mensurada a percepção de apoio social antes e depois da intervenção, usando o Questionário MOS de Suporte Social Percebido Resultados: O efeito da intervenção foi estatisticamente significante $(\mathrm{p}<0,05)$ e cada um dos fatores que a compõem: apoio emocional, informacional, apoio instrumental e interação positiva afetiva. Conclusões: As intervenções com o uso de recursos audiovisuais são efetivas como apoio para pacientes; e aqui a televisão e a rádio são os médios pelos quais maior apropriação é encontrada nessa população.

Palavras-chave: apoio social, doença crónica, tecnologia, recursos audiovisuais.

\section{Introducción}

Para las personas diagnosticadas con una enfermedad crónica y para su familia ocurren cambios significativos en su cotidianidad, por lo que es importante mitigar cuanto ello implica mediante la adquisición de conocimientos y habilidades específicas en autocuidado. Igualmente, es necesario el apoyo social que ayude en las áreas emocional, afectiva y cognitiva. Esto implica la interacción continua con personas o grupos que fortalezcan el cuidado, la adaptación y el manejo de situaciones propias en el proceso de cronicidad. Bowling definió el apoyo social como un proceso interactivo entre la persona y la red social en la que se encuentre para conseguir ayuda instrumental, económica y emocional (1).

El apoyo social se refiere a la provisión de ayuda instrumental o expresiva proporcionada por la red social, la comunidad o las personas de confianza del individuo tanto en situaciones cotidianas como de crisis que se relacionan con la calidad de vida, según Sarason, Sarason, Hacker y Basham, citados en Aranda y Pando (2).

En la visión de Rodríguez y Cohen y Seeman, citados en Baptista et al. (3), existen diferentes tipos de apoyo que una persona puede recibir de la red social, de los cuales tres son los más ampliamente estudiados: apoyo emocional (percibido como expresión de cariño, cuidados y preocupación), apoyo instrumental (ayudas prácticas que pueden ser dadas a otras personas) y apoyo informacional (nociones indispensables que permitirán que el individuo pueda guiar y orientar sus acciones en el momento de resolver problemas o tomar decisiones).

Este estudio concibe el apoyo social como la disponibilidad de una red social conformada por familiares, amigos, vecinos, personal de salud e instituciones, que llevan a la persona enferma crónica a percibir su efecto como una ayuda continua y comprometida, capaz de generar cambios positivos en las esferas conductual y emocional; este apoyo puede ser presencial o mediado por tecnología. El uso de tecnología de las comunicaciones por parte del profesional de enfermería y el equipo de salud tiene comprobado su valor como estrategia para proporcionar apoyo social a las personas con enfermedad crónica, cuidadores y familia (4).

Según las premisas anteriores, este estudio se diseñó para evaluar el efecto en la percepción de apoyo social y sus factores (emocional informacional, interacción positiva afectiva e instrumental) de la intervención Alguien apoya al que cuida en un grupo de enfermos crónicos usuarios de consulta externa de una institución prestadora de salud de tercer nivel. Su importancia radica en la poca literatura disponible sobre investigaciones centradas en determinar la percepción de apoyo social mediada por la tecnología en la persona con enfermedad crónica. 


\section{Materiales y método}

Estudio de tipo cuantitativo-preexperimental, con medición antes de la intervención y después de esta. La población correspondió a una muestra de 116 personas con enfermedad crónica vinculadas al Programa de Paciente Crónico del Hospital Universitario de la Samaritana-Unidad Funcional-Girardot, vinculadas durante el segundo semestre de 2014 que formaran parte del grupo experimental. La selección de la muestra fue por conveniencia, de una población que cumplía con los siguientes criterios de inclusión: pertenecer al Programa de Paciente Crónico, llevar en seguimiento clínico durante al menos seis meses y no cursar con deterioro cognitivo. Una vez comunicado y firmado el consentimiento informado, se caracterizaron las personas con enfermedad crónica, aplicando la ficha Caracterización de la Persona con Enfermedad Crónica o GCPC-UN-P, desarrollada por el Grupo de Investigación Cuidado de Enfermería Paciente Crónico de la Facultad de Enfermería de la Universidad Nacional de Colombia, donde se consignaron las características sociodemográficas, condiciones para el cuidado, percepción de carga y nivel de apropiación de las tecnologías de la información y la comunicación (TIC) que tienen las personas con enfermedad crónica.

La percepción del cambio en el apoyo social se determinó con la aplicación del cuestionario MOS de Soporte Social Percibido, antes de la intervención y después de esta. Dicho instrumento tiene pruebas psicométricas validadas para Colombia (5). El análisis factorial del instrumento en Colombia reconoce tres factores: emocional informacional, instrumental e interacción positiva afectiva, con un alfa de Cronbach 0,93, 0,79 y 0,86 , respectivamente. Las dimensiones reportadas en este análisis son la de apoyo emocional/ informacional, que agrupa los ítems 3, 4, 8, 9, 13, 14, 16, 17 y 19; apoyo instrumental, que agrupa los ítems 2, 5, 12 y 15, y la dimensión interacción positiva/afectiva, que agrupa los ítems 6, 7, 10,11, 18 y 20.

La estrategia de intervención está diseñada con cuatro sesiones para cada uno de los cuatro grupos conformados por cerca de 30 personas. El primer taller se orienta al apoyo emocional informacional con la proyección del video Alguien apoya al que cuida (6), elaborado por el Grupo Cuidado de Enfermería al Paciente Crónico, que enmarca los tipos de apoyo social y los componentes fundamentales para el cuidado del paciente con enfermedad crónica y su cuidador. En el segundo taller se interviene la interacción positiva afectiva, utilizando como apoyo TIC los videos Sacúdete y sigue (7) y Som Sabadell flashmob (8). El tercer taller se enfoca en el apoyo instrumental, con presentaciones educativas en Microsoft Office Power Point, que orientan el cuidado de la persona con diabetes e hipertensión. Adicionalmente, se entregan folletos que informan sobre los principales cuidados de las personas que presentan estas situaciones de salud. En el cuarto taller se retoma el apoyo social con todos sus factores y nuevamente se presentaba el video Alguien apoya al que cuida. En cada taller se reflexionó con cada uno de los mensajes de los videos, se socializaron las respuestas dadas a preguntas pertinentes a cada tema, con el objetivo de resaltar la importancia de los tipos de apoyo disponibles para el cuidado de la persona con enfermedad crónica y el cuidador.

El análisis estadístico de los datos se realizó utilizando los programas Excel y SPSS 17.0. Para describir la tendencia de los datos antes de la intervención realizada y después de esta, se calcularon las medidas de tendencia central media y mediana y de dispersión la desviación estándar.

Durante la investigación se siguieron los parámetros éticos relacionados en la Resolución 8490 de 1993, que establece las normas científicas, técnicas y administrativas para investigación en salud, que le garantiza al sujeto de investigación el respeto a su dignidad, la protección de sus derechos y asegura el bienestar de la persona con enfermedad crónica. El consentimiento informado, la participación voluntaria, el manejo confidencial de la información recolectada y la autorización expresa del Comité de Investigación del Hospital Universitario de la Samaritana formaron parte del diseño y desarrollo del trabajo. La investigación también se acogió a la Ley 266 de 1996, por la cual se reglamenta el ejercicio de la profesión de enfermería en Colombia, consagra los principios éticos y morales del profesional de enfermería que deben tenerse en cuenta para la realización de actividades de enfermería. 


\section{Resultados}

\section{Caracterización de las personas con enfermedad crónica}

La muestra analizada estuvo conformada por 116 personas con edades comprendidas entre los 54 y los 67 años. El 83\% de la muestra corresponde a mujeres con un promedio de edad de 64,8 $\pm 10,3$ años, y en los hombres la edad promedio es de 62,5 $\pm 10,3$ años. El 95\% de los pacientes se encuentra en los estratos socioeconómicos 1 y 2 y el nivel de escolaridad más frecuente en los pacientes es la primaria incompleta, con el 38,7\% de la muestra.

Los estados civiles más frecuentes en la muestra son unión libre (25\%) y viudo (24,14\%). Los pacientes casados constituyen el 19\% de la muestra, y los separados, el 7,8\%. Al reportar su ocupación, el 74,1\% indica que se dedica a las labores del hogar (69\% mujeres y 5\% hombres), el 21,6\% son trabajadores independientes y el 4,3\% son empleados.

El $74,1 \%$ de la muestra analizada está conformada por pacientes hipertensos. Los pacientes con diagnóstico único de diabetes representan el $12,9 \%$ de la muestra y el $1 \%$ restante presenta otras patologías crónicas.

\section{Autovaloración en el uso de las tecnologías de la información}

Los resultados muestran que los pacientes con enfermedad crónica tienen niveles de conocimiento principalmente alto y medio para el uso de la televisión, el radio y el teléfono; en contraste, más del 90\% de la muestra manifiesta tener un nivel bajo de conocimiento en el uso del computador e internet (figura 1).

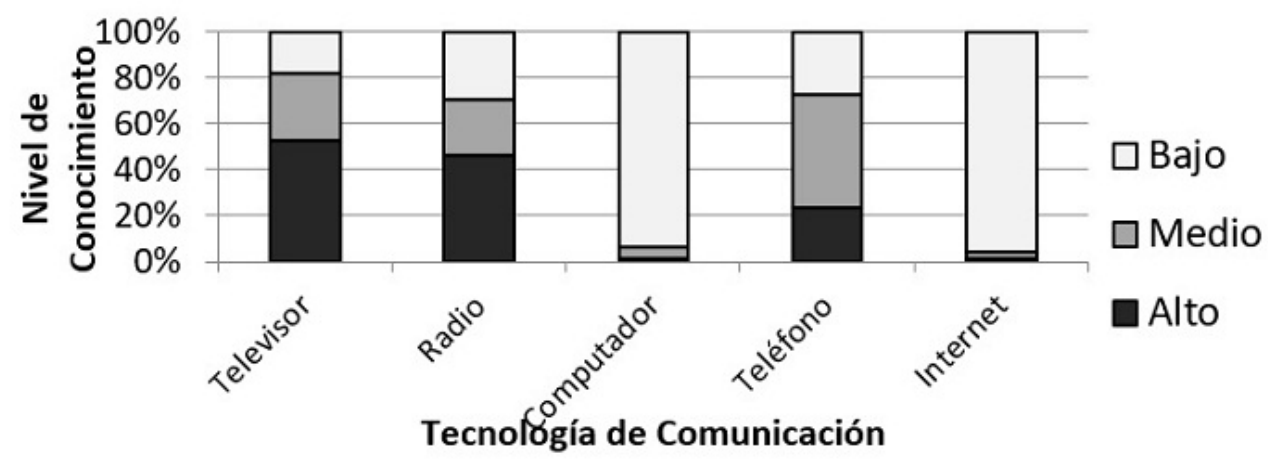

FIGURA 1.

Autovaloración de los pacientes con enfermedad crónica en el uso de las TIC Fuente: elaboración propia

Como parte de la autovaloración en el uso de las TIC, se indagó por la posibilidad de acceso a estas tecnologías como apoyo en el cuidado de la salud. El resultado obtenido muestra una tendencia similar a la del nivel de conocimiento, con la radio y la televisión como los medios de comunicación con mayor posibilidad de acceso para este grupo de pacientes (figura 2). 


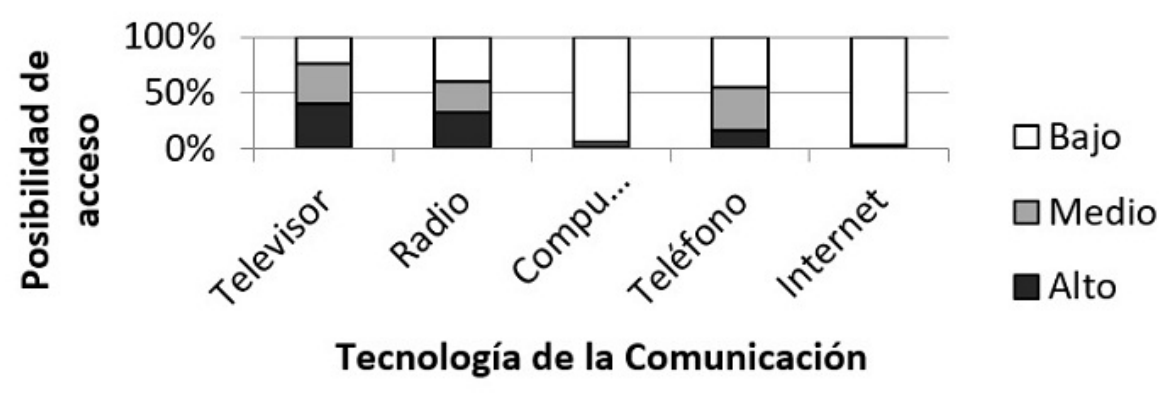

FIGURA 2.

Posibilidad de acceso a las tecnologías de comunicación para apoyar el cuidado de los pacientes con enfermedad crónica

Fuente: elaboración propia

La tendencia citada anteriormente se repite al indagar por la frecuencia de uso de las diferentes tecnologías. El uso más frecuente (nivel alto) se observó en televisión, radio y teléfono.

\section{Apoyo social percibido por los pacientes con enfermedad crónica antes de la intervención realizada con el uso de TIC y después de esta}

El Cuestionario MOS de Apoyo Social Percibido integra tres factores: el apoyo emocional informacional, el apoyo instrumental y la interacción positiva afectiva. En el análisis estadístico descriptivo general y por factores se observó un incremento en la media de apoyo percibido por los pacientes con enfermedad crónica después de la intervención realizada.

El análisis inferencial usando la prueba pareada de Wilcoxon muestra que el incremento observado en las medianas (tabla 1) es estadísticamente significativo en todas las comparaciones del soporte social percibido, y sus factores, antes y después de la intervención, ya que los valores de p obtenidos son menores al valor de alfa establecido de 0,05 ( $95 \%$ de confianza).

TABLA 1.

Análisis descriptivo e inferencial (Prueba de Wilcoxon) de la tendencia en el apoyo social percibido y sus factores antes de la intervención realizada con el uso de TIC y después

\begin{tabular}{lrrrrrrrr}
\hline & \multicolumn{2}{c}{ Apoyo social } & \multicolumn{2}{c}{$\begin{array}{c}\text { Emocional } \\
\text { informacional }\end{array}$} & Instrumental & \multicolumn{2}{c}{$\begin{array}{c}\text { Interacción } \\
\text { positiva afectiva }\end{array}$} \\
\hline & Pre & Post & Pre & Post & Pre & Post & Pre & Post \\
\hline Media & 75,5 & 79,6 & 35 & 37,3 & 15,6 & 16,4 & 24,9 & 25,9 \\
\hline Mediana & 79,5 & 86 & 38 & 40 & 17 & 18 & 27 & 28 \\
\hline Desviación estándar & 18,1 & 16,5 & 9,3 & 8,7 & 4,5 & 4,3 & 5,9 & 5,2 \\
\hline Mínimo & 19 & 19 & 9 & 9 & 4 & 4 & 6 & 6 \\
\hline Máximo & 95 & 95 & 45 & 45 & 20 & 20 & 30 & 30 \\
\hline Prueba de Wilcoxon (p) & 6,97 & E-05 & 0,000122 & & 0,014 & & 0,017 \\
\hline
\end{tabular}

Fuente: elaboración propia

En todas las comparaciones realizadas se puede observar que las medidas de tendencia central, media y mediana aumentaron después de la intervención y que la variabilidad de los datos, representada en la desviación estándar, se redujo. Gráficamente, la anterior tendencia puede visualizarse en los gráficos de caja y bigotes (figura 3). 

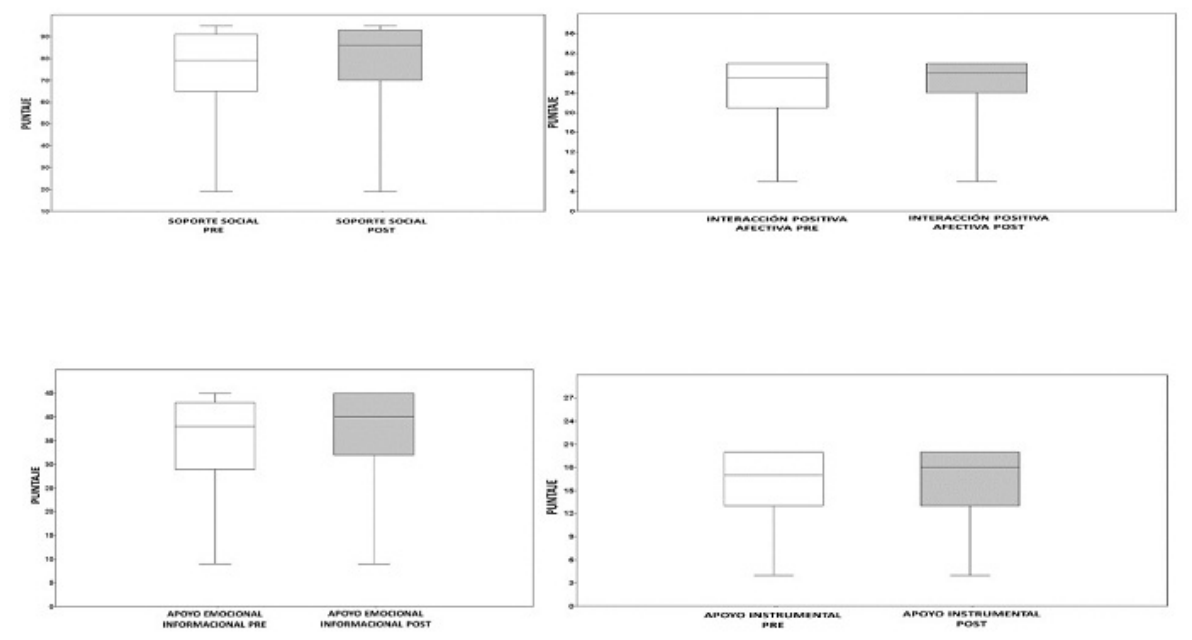

FIGURA 3.

Cambio en la percepción de apoyo social y sus factores en pacientes con enfermedad crónica antes de la intervención con el uso de TIC y después Fuente: elaboración propia

\section{Discusión}

Después de la intervención mediada por recursos audiovisuales hubo cambios estadísticamente favorables sobre la percepción de apoyo social en los pacientes, y ello lleva a que el profesional de enfermería adquiera el reto de innovar en la práctica y en las instituciones de salud como una necesidad de promover espacios para el desarrollo de intervenciones innovadoras (9).

En lo que respecta a esta investigación, cerca de la mitad de los adultos mayores se encuentran casados o en unión libre, estados que se asocian con un mejor acompañamiento y apoyo, resultados hallados por Zapata et al. (10) y Vinaccia et al. (11), quienes aseveran que contar con una unión de pareja constituye una fortaleza afectiva para el adulto mayor. La revisión del estudio de Baptista et al. destaca la importancia del apoyo familiar y social al concluir que actúa como "amortiguador frente a las dificultades encontradas por los miembros de una determinada familia" (3). Así, la red de apoyo social donde la familia, los amigos, el personal de salud, los compañeros de trabajo son los principales proveedores del apoyo emocional cuando se presentan eventos devastadores puede servir de amortiguador de los efectos de las emociones negativas creados por la enfermedad (12,13).

Al comparar los resultados de esta investigación con un estudio realizado en el área metropolitana del Valle de Aburrá (Colombia) con 14 mujeres diagnosticadas y tratadas de cáncer de cuello uterino, se identificaron similitudes en el apoyo informacional satisfactorio en relación con los profesionales de enfermería: aquellos se mostraron amables y comprensivos, al promover un diálogo que posibilitaba información y apoyo, lo que fue un bálsamo en medio del sufrimiento (13). Otro estudio demostró que existe una fuerte correlación entre los tres tipos de apoyo social, donde el afrontamiento conductual tuvo un mayor impacto en el bienestar subjetivo (14).

En relación con el apoyo tecnológico, en este estudio la radio y la televisión son los de mayor disponibilidad y, paralelamente, son los que los usuarios manejan con más habilidad, lo que respaldó el uso de recursos audiovisuales como el video y presentaciones educativas en la intervención, para modificar la percepción de apoyo, sustentado por Dalmolin et al. (15), Santana et al. (16) y Campos et al. (17), quienes expresan que la aplicabilidad de la tecnología audiovisual desarrollada ha abierto nuevas posibilidades de formación a 
los pacientes y complementa las orientaciones educativas, al posibilitar transformar y repensar las prácticas pedagógicas en enfermería y multidisciplinarias.

En un centro de salud en Perú, los adultos mayores reciben ayuda material directa o servicios por parte de la red donde se encuentran, haciendo uso de esta para conseguir objetivos y metas (18). Así, el apoyo instrumental dado por folletos, ayuda directa en casa, videos y audios son una alternativa educativa en el abordaje para el autocuidado, el incremento de las tasas de adherencia y efectividad de los tratamientos farmacológicos instaurados y la disminución de complicaciones y aparición de comorbilidades relacionadas con su enfermedad base (19).

Por otro lado, hay un escaso conocimiento sobre el uso del computador y, adicionalmente, la accesibilidad a esta ayuda es muy limitada tanto en el aparato como de internet para habilitar su uso. Estos hallazgos coinciden con otros estudios (18) y deben servir de base para que las instituciones de salud en la región diseñen las ayudas dirigidas a esta población, pensando en llegar de la manera más efectiva al mayor número de usuarios. De acuerdo con un estudio realizado por Barrera et al. (20), la herramienta tecnológica de mayor uso para proveer apoyo social fue internet, seguido por el teléfono y la combinación del teléfono e internet.

Según lo expresado por Villalobos (18), el apoyo afectivo se refiere a las expresiones de afecto y amor que les son ofrecidas a los individuos por parte de su grupo más cercano, que generalmente es la familia. Consideramos que el cambio menos significativo en la percepción de este apoyo en las personas con enfermedad crónica estudiadas es debido probablemente a que no fue suficiente el tiempo de interacción por medio de los talleres. Esta dimensión, que está relacionada con manifestaciones de sentirse valorado, estimado, amado y cuidado como ser humano, dentro del grupo familiar y social, requiere un fortalecimiento de los vínculos afectivos y una continuidad en la intervención con valoraciones periódicas que aseguren un cambio en su percepción. Como resultado en el estudio de Alpi y Quiceno la afectividad se destacó como el área que evalúa la función familiar (21).

En cada uno de los talleres se tuvieron en cuenta las necesidades espirituales del grupo de pacientes, por lo que se realizó una actividad exclusiva sobre el cuidado espiritual y su importancia para el cambio de actitud hacia la vida, a pesar de la adversidad (22,23). De acuerdo con Chiang y Lee (24), los humanos tienen cuatro necesidades espirituales durante la enfermedad y la salud: significado y propósito en la vida, dar y recibir amor, esperanza y creatividad y perdón.

El apoyo social puede ser facilitado por un sistema formal o informal (9); de ahí que los proveedores de salud sean parte del sistema formal, con una red organizada de apoyo donde sean necesarias las demostraciones de afecto y cercanía durante la provisión del cuidado.

\section{Conclusiones}

La implementación de una intervención con recursos audiovisuales produjo un cambio estadísticamente significativo del apoyo emocional/informacional, apoyo instrumental y también en la interacción positiva afectiva.

El menor efecto estuvo en la interacción positiva afectiva; tal vez por estar más ligado a demostraciones de amor, cariño, afecto y empatía con personas realmente cercanas.

La intervención transmitida a través de recursos audiovisuales favoreció la dinámica de grupo, creó un ambiente de confianza y permitió la socialización de vivencias de acuerdo con la situación de salud experimentada.

Para el profesional de enfermería comprometido con el cuidado de la persona con enfermedad crónica, el uso de la tecnología y los recursos audiovisuales constituyen uno de los pilares que facilitan el acercamiento y comprensión de la información necesarios para modificar la percepción de apoyo. 


\section{Referencias}

1. Fachado AA, Menéndez M, González L. Apoyo social: Mecanismos y modelos de influencia sobre la enfermedad crónica. Cad Aten Primaria [Internet]. 2013 [citado 2015 marzo 20];19:118-23. Disponible en: https://www.a gamfec.com/wp/wpcontent/uploads/2014/07/19_2_ParaSaberDe_3.pdf

2. Aranda C, Pando M. Conceptualización del apoyo social y las redes de apoyo social. Rev Investig Psicol [Internet]. 2013 [citado 2015 abril 22];16(1):233-45. Disponible en: https://www.revistasinvestigacion.unmsm.edu.pe/in dex.php/psico/article/view/3929

3. Baptista MN, Rigotto DM, Cardoso HF, Rueda FJ. Soporte social, familiar y autoconcepto: relación entre los constructos. Psicol Caribe [Internet]. 2012 [citado 2016 abril 24];29(1):1-18. Disponible en: https://www.sci elo.org.co/pdf/psdc/v29n1/v29n1a02.pdf

4. Vega OM, Gonzáles DS. Soporte social mediante intervención telefónica en cuidadores de enfermos crónicos discapacitados en Cúcuta, Colombia. Respuestas [Internet]. 2013 [citado 2016 junio 5];18(1):32-42. Disponible en: https://www.revistas.ufps.edu.co/ojs/index.php/respuestas/article/view/384

5. Londoño AN, Rogers HL, Castilla TJ, Posada GS, Ochoa AN, Jaramillo PM, et al. Validación en Colombia del cuestionario MOS de Apoyo Social. Int J Psychol Res. 2012;5(1):142-50.

6. Universidad Nacional de Colombia. Alguien apoya al que cuida [video]. 2014 marzo 27 [citado 2014 enero 7]. [13:33]. Disponible en: https://www.youtu.be/CU9wKZVooUk

7. Osorio M. Reflexiones de vida-sacúdete y sigue [video]. 2011 Abril 6 [citado 2014 jun 3].[2:39]. Disponible en: h ttps://www.youtu.be/5_2LtHMM2sE

8. Banco Sabadell. Som Sabadell flashmob [video]. 2012 mayo 31 [citado 2014 jun 3].[05:40]. Disponible en: https: //www.youtu.be/GBaHPND2QJg.

9. Garizábalo C, Rodríguez A, Cañón W. Soporte social enfocado a personas con diabetes: una necesidad desde enfermería. Revista Cuidarte [Internet]. 2018 Dec 2 [citado 2019 ene 3];10:697. Disponible en: https://www. revistacuidarte.udes.edu.co/index.php/cuidarte/article/view/697/1060

10. Zapata BI, Delgado NL, Cardona D. Apoyo social y familiar al adulto mayor del área urbana en Angelópolis, Colombia. Rev Salud Pública. 2015;17(6):848-60.

11. Vinaccia S, Quiceno J, Lozano F, Romero S. Calidad de vida relacionada con la salud, percepción de enfermedad, felicidad y emociones negativas en pacientes con diagnóstico de artritis reumatoide. Acta Colomb Psicol [Internet]. 2017 [citado 2017 jun 15];20(1):49-59.

12. Vanegas OJ, Krause JM, Bauer S, Moessner M, Espinosa D, Carrasco GA, et al. Uso de e-mental health para el seguimiento posterapia de pacientes con depresión en Colombia. Rev Virtual Univ Catol Norte [Internet]. 2017 [citado 2018 feb 13];(52):41-56. Disponible en: https://www.revistavirtual.ucn.edu.co/index.php/RevistaUC $\mathrm{N} /$ article/view/943/1390

13. Yasmeen B, Khan MZ, Jamshaid N, Salman M, Abbas S. Heart and kidney patients; correlational patterns of social support with coping strategies and subjective well-being. Profess Med J. 2015 [citado 2018 feb 13];22(2):235-43.

14. Castellano CL. La influencia del apoyo social en el estado emocional y las actitudes hacia la vejez y el envejecimiento en una muestra de ancianos. Rev Int Psicol Ter Psicol. 2014;14(3):365-77.

15. Dalmolin A, Oliveira N, Coppetti L, Rossato G, Sonego J, Nunes da Silva ME. Educational video as a healthcare education resource for people with colostomy and their families. Rev Gaucha Enferm [Internet]. 2016 Dec 2 [citado 2019 ene 3];37:1-9. Disponible en: https://www.search.ebscohost.com.proxy.unisanitas.edu.co:90/logi n.aspx?direct $=$ true $\& \mathrm{db}=\mathrm{c} 8 \mathrm{~h} \& \mathrm{AN}=127492089 \&$ lang=es\&site $=$ ehost-live

16. Santana M, Aguirremota M, Lodeiro H, González M, Castro S, Escudero J. Resultados de un programa educativo con refuerzo audiovisual durante la sesión, en pacientes en hemodiálisis. Enferm Nefrol [Internet]. 2017 abr [citado 2019 ene 3];20(2):126-31. Disponible en: https://www.search.ebscohost.com.proxy.unisanitas.edu.co: 90/login.aspx?direct $=$ true\& $\mathrm{db}=\mathrm{c} 8 \mathrm{~h} \& \mathrm{AN}=123919411$ \&lang=es\&site=ehost-live

17. Campos E, Neroni A, Thaís M, Garbin L, Merizio B, Moreli L. Efeito de vídeo educativo no comportamento de higiene bucal de pacientes hematológicos. Rev Eletronica Enferm [Internet]. 2014 abr [citado 2019 ene 
3];16(2):304-11. Disponible en: https://www.search.ebscohost.com.proxy.unisanitas.edu.co:90/login.aspx?dir ect $=$ true \&db=c8h\&AN=109817936\&lang=es\&site=ehost-live

18. Villalobos CE. Apoyo social en los adultos mayores de un Centro de Salud de José Leonardo Ortiz [Tesis de pregrado]. Perú: Universidad Católica Santo Toribio de Mogrovejo; 2015.

19. Molina D, Valencia S, Agudelo L. La educación a pacientes y su corresponsabilidad como herramientas terapéuticas. Rev Colomb Cardiol [Internet]. 2017 abr [citado 2019 ene 03];24(2):176-81. https://dx.doi.org/10.1016/j.rc car.2016.07.013

20. Barrera L, Carrillo GM, Chaparro L, Pinto N, Sánchez B. Soporte social con el uso de TIC's para cuidadores familiares de personas con enfermedad crónica. Rev Salud Pública. 2011;13(3):446-57.

21. Alpi SV, Quiceno JM. Calidad de vida relacionada con la salud y enfermedad crónica: estudios colombianos. Av Disciplina. 2012;6(1):123-36.

22. Rivera LA, López LM. Clinical practice and spirituality. Ann Psychol [Internet]. 2007 [citado 2018 jun 7];23(1):125-36. Disponible en: https://www.revistas.um.es/analesps/article/view/23111/22391

23. Petrie C. Spirituality: part of nursing practice, but too often neglected. Am J Nurs. 2016;116(9):19-20.

24. Chiang YC, Lee HC, Chu TL, Han CY, Hsiao YC. The impact of nurses' spiritual health on their attitudes toward spiritual care, professional commitment, and caring. Nurs Outlook. 2016;64(3):215-24.

\section{Notas}

* Artículo de investigaciónLimitaciones del estudio: como fue una investigación preexperimental, no se contó con un grupo control para la comparación; por lo tanto, se recomienda en próximos estudios un diseño con casos y controles para una mejor evaluación.

1. Financiamiento: Universidad de Cundinamarca

2. Conflictos de interés: los autores declaran no tener conflictos de interés.

\section{Licencia Creative Commons CC BY 4.0}

Cómo citar este artículo: Hernández Bustos A, Rojas Martínez MV, Cuevas Rodríguez GN. La autopercepción del apoyo social del paciente con enfermedad crónica, mediado por tecnología. Investig Enferm Imagen Desarr. 2020;21(2). https://doi.org/10.11144/Javeriana.ie21-2.aasp 